\title{
Solución numérica de la ecuación fundamental \\ de Solow \\ con función de producción CES*
}

\section{Numerical Solution of the \\ Equation of Fundamental Solow \\ CES Production Function}

Nicolás Marciales Parra**

Recibido: 26 de noviembre de 2012

Aprobado: 27 de Febrero de 2013

\section{Resumen}

En este trabajo se aborda una alternativa para la solución de la ecuación fundamental de Solow, en el caso que la función de producción es una función CES. Se mostrará cómo cambiar la función de producción altera substancialmente la ecuación fundamental y los procesos de análisis alrededor de ella. Es por esto que se plantea la forma

* Cómo citar este artículo: Marciales, N. (2013). Solución numérica de la ecuación fundamental de Solow con función de producción CES. Revista CIFE, 15 (23), pp. 77-91.

* Matemático Universidad Nacional de Colombia, Mg. en Ciencias Económicas Universidad Santo Tomás Profesor de la Universidad Santo Tomás. Correo: nicolasmarciales@usantotomas.edu.co 
ISSN: 0124-3551 / Año 15, No 23 / julio-diciembre / pp. 77-91

de cómo resolver la ecuación fundamental de Solow por el método de Euler, el cual determina aproximaciones numéricas de la solución de una ecuación diferencial de primer orden.

Palabras clave: CES, Método de Euler, Métodos Numéricos, Modelo de crecimiento de Solow.

Clasificación JEL: C63, O40

\section{Abstract}

This work approach an alternative for the solution of Solow's fundamental equation for the case of CES output function. Changing the output function in this equation implies a modification in the analysis of the equation. It used the Euler's method to solve the Solow's fundamental equation in the model, a numerical method to solve ordinary first -order differential equations.

Keywords: CES, Euler's method, Numerical Methods, Solow Growth Model.

Classification JEL: C63, O40 


\section{Introducción}

El crecimiento económico busca explicar el comportamiento de la economía de largo plazo y lograr entender la razón por la cual algunos países son más ricos que otros. Dentro de los modelos de crecimiento endógenos se encuentra el modelo de Solow Swan, el cual es la base para otros modelos de crecimiento. El modelo neoclásico de Solow - Swan busca explicar el crecimiento de la economía que crece con base en el capital físico (Sala - i - Martin, 2000).

En la versión más simple del modelo (sin gasto del gobierno, economía cerrada, tasa de crecimiento poblacional constante, tasa de depreciación constante, entre otras) Se obtiene la ecuación diferencial:

$$
k=s f(k)-(n+\delta) k
$$

Donde es el capital per cápita (o relación capital - trabajo), $f(k)$ es una función de producción, corresponde a la propensión marginal al ahorro, con y tasas de crecimiento poblacional de tasa de depreciación del capital. La notación $k$ corresponde $\frac{d k}{d t}$. Esta ecuación diferencial de primer orden recibe el nombre de ecuación fundamental de Solow.

Suponiendo que la producción de los bienes se hace mediante la combinación de trabajo $L$, capital físico $K$, y tecnología $A$, se puede afirmar que:

$$
\Upsilon=F(A, L, K)
$$

Y que adicionalmente suponiendo $A$ constante se obtiene una función de producción de la forma:

$$
\Upsilon=F(L, K)
$$

Dentro de la ecuación fundamental de Solow, subyacen supuestos adicionales sobre la función de producción. Estos supuestos son importantes para el éxito del modelo y que, aunque pueden parecer de difícil cumplimiento, se emplean para simplificar el mismo.

Los supuestos de buen comportamiento de la función de producción neoclásica son ${ }^{1}$ :

1. Homogénea de grado uno.

2. Cóncava.

3. Condiciones de Inada:

$$
\lim _{L \rightarrow \infty} \frac{\partial F}{\partial L}=\lim _{K \rightarrow \infty} \frac{\partial F}{\partial K}=0 ;
$$

1 Otras formas equivalentes de los supuestos de la función de producción se encuentran en Romer(1996). 


$$
\lim _{L \rightarrow 0} \frac{\partial F}{\partial L}=\lim _{K \rightarrow 0} \frac{\partial F}{\partial K}=\infty
$$

La función de producción neoclásica más conocida que cumple estos supuestos es la función Cobb - Douglas definida de la forma:

$$
F(L, K)=A L^{1-\alpha} K^{\alpha} ; 0<\alpha<1
$$

$\mathrm{Al}$ convertir la función en términos per cápita, convierte la ecuación fundamental de Solow en:

$$
\stackrel{*}{k}=s A k^{\alpha}-(n+\delta) k
$$

La cual es una ecuación diferencial de Bernoulli².

Desde una primera perspectiva, se desea examinar el valor del capital de estado estacionario $k$, valor donde la curva de ahorro y la curva de depreciación son iguales. Sin embargo, la solución de la ecuación diferencial permite conocer cuál es el valor del capital en cualquier instante del tiempo antes de llegar a un estado estacionario, teniendo presente que:

$$
\lim _{t \rightarrow \infty} k(t)=k^{*}
$$

\section{La función de producción CES en el modelo de Solow - Swan}

Cuando se piensa en funciones de producción más generales, podrían o no cumplirse las condiciones para las cuales se llegue a un estado estacionario en la economía. Pero el problema se complica aún más si se desea obtener una solución analítica de la ecuación diferencial.

El caso más destacado es cuando se tiene la función CES, definida por:

$$
F(L, K)=\left(\alpha L^{\rho}+(1-\alpha) K^{\rho}\right)^{1 / \rho}
$$

Donde $0<\alpha<1$, y $\rho<1$ son parámetros de la función. El parámetro $\rho$ representa el nivel de sustitución entre las variables. Cuando la función toma una forma Cobb-Douglas. Si $\rho \rightarrow-\infty$, la función toma una forma Leontieff.

Esta función satisface condiciones de buen comportamiento:

2 Una ecuación diferencial es Bernoulli si tiene la forma: $y^{\prime}+p(x) y=f(x) y^{\mathrm{n}}$. Suponiendo que $y=y(x) \cdot \mathrm{y}$ 
Homogénea de grado uno:

$$
\begin{gathered}
F(\lambda L, \lambda K)=\left(\alpha(\lambda L)^{\rho}+(1-\alpha)(\lambda K)^{\rho}\right)^{1 / \rho}=\left(\lambda^{\rho}\left(\alpha L^{\rho}+(1-\alpha) K^{\rho}\right)\right)^{1 / \rho} \\
=\lambda F(L, K) \\
y^{\prime}+p(x) y=f(x) y^{n} .
\end{gathered}
$$

Cóncava:

$$
\begin{gathered}
\frac{\partial F}{\partial L}=L^{-1+\rho} \alpha\left(K^{\rho}(1-\alpha)+L^{\rho} \alpha\right)^{-1+\frac{1}{\rho}}>0 \\
\frac{\partial F}{\partial K}=K^{-1+\rho}(1-\alpha)\left(K^{\rho}(1-\alpha)+L^{\rho} \alpha\right)^{-1+\frac{1}{\rho}}>0 \\
\frac{\partial^{2} F}{\partial L^{2}}=\frac{K^{\rho} L^{-2+\rho}(-1+\alpha) \alpha\left(K^{\rho}(1-\alpha)+L^{\rho} \alpha\right)^{\frac{1}{\rho}}(-1+\rho)}{\left(-K^{\rho}+K^{\rho} \alpha-L^{\rho} \alpha\right)^{2}}<0
\end{gathered}
$$

Siempre que:

$$
-(-1+\alpha)(-1+\rho)<0 \quad \rho<1
$$

Análogamente:

$$
\frac{\partial^{2} F}{\partial L^{2}}=\frac{K^{\rho} L^{-2+\rho}(-1+\alpha) \alpha\left(K^{\rho}(1-\alpha)+L^{\rho} \alpha\right)^{\frac{1}{\rho}}(-1+\rho)}{\left(-K^{\rho}+K^{\rho} \alpha-L^{\rho} \alpha\right)^{2}}<0
$$

Siempre que:

$$
-(-1+\alpha)(-1+\rho)<0 \quad \rho<1
$$

Adicionalmente se tiene que el determinante de la matriz hessiana para la CES corresponde a:

$$
\operatorname{Det}\left(\begin{array}{l}
-\frac{K^{\rho} L^{-2+\rho}(-1+\alpha) \alpha\left(K^{\rho}(1-\alpha)+L^{\rho} \alpha\right)^{\frac{1}{\rho}}(-1+\rho)}{\left(-K^{\rho}+K^{\rho} \alpha-L^{\rho} \alpha\right)^{2}} \frac{K^{-1+\rho} L^{-1+\rho}(-1+\alpha) \alpha\left(K^{\rho}(1-\alpha)+L^{\rho} \alpha\right)^{\frac{1}{\rho}}(-1+\rho)}{\left(-K^{\rho}+K^{\rho} \alpha-L^{\rho} \alpha\right)^{2}} \\
\frac{K^{-1+\rho} L^{-1+\rho}(-1+\alpha) \alpha\left(K^{\rho}(1-\alpha)+L^{\rho} \alpha\right)^{\frac{1}{\rho}}(-1+\rho)}{\left(-K^{\rho}+K^{\rho} \alpha-L^{\rho} \alpha\right)^{2}} \frac{K^{-2+\rho} L^{\rho}(-1+\alpha) \alpha\left(K^{\rho}(1-\alpha)+L^{\rho} \alpha\right)^{\frac{1}{\rho}}(-1+\rho)}{\left(-K^{\rho}+K^{\rho} \alpha-L^{\rho} \alpha\right)^{2}}
\end{array}\right)
$$


Y gracias a que la función es $C^{2}$, la función es cóncava (mas no estrictamente cóncava) (Sydsaeter, Strom, \& Berk, 2005).

\section{Condiciones de Inada ${ }^{3}$ :}

$$
\begin{aligned}
& \lim _{L \rightarrow 0} L^{-1+\rho} \alpha\left(K^{\rho}(1-\alpha)+L^{\rho} \alpha\right)^{-1+\frac{1}{\rho}} \\
& =\lim _{L \rightarrow \infty} \alpha\left(\frac{K^{\rho}(1-\alpha)}{L^{\rho}}+\alpha\right)^{-1+\frac{1}{\rho}}=\infty \\
& \lim _{L \rightarrow \infty} L^{-1+\rho} \alpha\left(K^{\rho}(1-\alpha)+L^{\rho} \alpha\right)^{-1+\frac{1}{\rho}} \\
& =\lim _{L \rightarrow \infty} \alpha\left(\frac{K^{\rho}(1-\alpha)}{L^{\rho}}+\alpha\right)^{-1+\frac{1}{\rho}}=0
\end{aligned}
$$

Para escribir la ecuación fundamental de Solow en términos de la relación capital trabajo, es requerido que la función de producción sea homogénea de grado 1. De esta forma, con la función GES se obtiene:

$$
f(k)=\frac{F(L, K)}{L}=\frac{\left(\alpha L^{\rho}+(1-\alpha) K^{\rho}\right)^{\frac{1}{\rho}}}{L}=\left(\alpha+\frac{(1-\alpha) K^{\rho}}{L^{\rho}}\right)^{1 / \rho}
$$

De lo que se obtiene que la función per cápita corresponde a:

$$
f(k)=\left(\alpha+(1-\alpha) k^{\rho}\right)^{1 / \rho}
$$

Y por tanto, la ecuación fundamental de Solow resultante es:

$$
\stackrel{*}{k}=s A\left(\alpha+(1-\alpha) k^{\rho}\right)^{1 / \rho}-(n+\delta) k
$$

En el análisis de estado estacionario, se quiere observar si la curva de ahorro y la curva de depreciación se cortan en un punto, el cual corresponde al capital de estado estacionario. Dado que la curva de ahorro depende de los parámetros adicionales de $\alpha$ y $\rho$, se muestran a continuación algunos casos. Las gráficas fueron elaboradas en Mathematica ${ }^{\circledR}$ con valores en los parámetros tomados arbitrariamente: $s=0,3 ; \delta=0,1 ; n=0,015 ; A=1 ; \alpha=0,5$, observando diferenciales en el parámetro $\rho$ : 
Figura 1. Curva de ahorro y depreciación cuando $\rho=0,6$

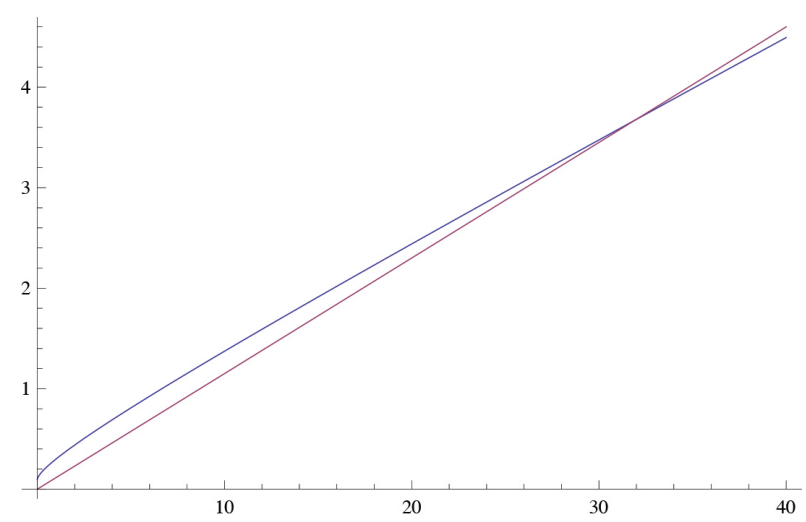

Figura 2. Curva de ahorro y depreciación cuando $\rho=0,1$

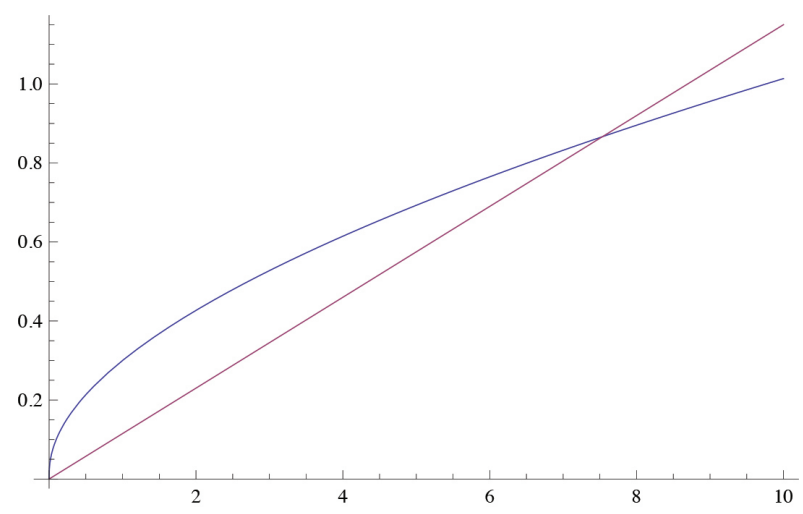

Figura 3. Curva de ahorro y depreciación cuando $\rho=-2$

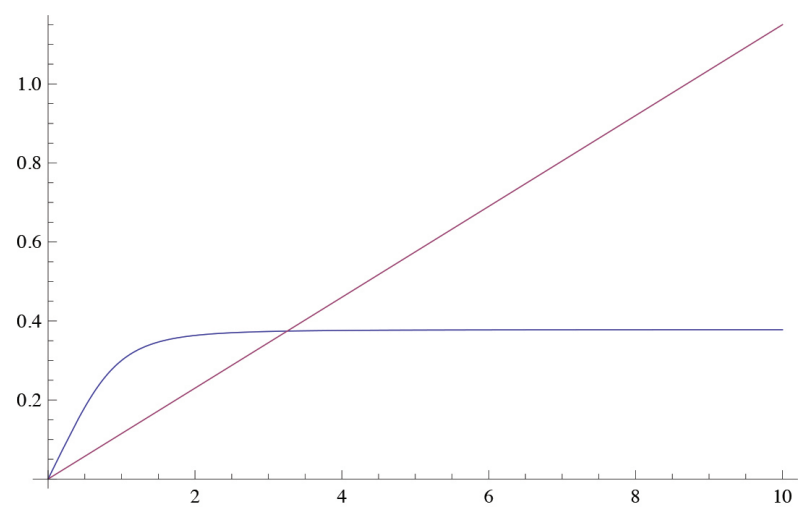




\section{Solución de la ecuación fundamental}

Dependiendo de los parámetros del problema, y suponiendo que puede no determinarse el valor del capital de estado estacionario. Pero ello conlleva a la verificación de la existencia de una solución real positiva de la ecuación algebraica resultante. Pero el panorama es aún más desalentador cuando se desea examinar cómo es la solución de la ecuación diferencial.

Como se mencionaba anteriormente, si la función de producción es Cobb - Douglas, la ecuación fundamental de Solow es una ecuación diferencial de Bernoulli, cuya solución se obtiene mediante la sustitución $\mathrm{z}=k^{1-\alpha}$. Esto conlleva a que la solución de la ecuación fundamental es:

$$
k(t)=\left(\frac{s A}{n+\delta}+c e^{-(1-\alpha)(n+\delta) t}\right)^{1 /(1-\alpha)}
$$

A pesar que la forma de la ecuación fundamental de Solow es una ecuación diferencial de variables separables:

$$
\begin{gathered}
k^{*}=s f(k)-(n+\boldsymbol{\delta}) k \\
\frac{d k}{d t}=s f(k)-(n+\boldsymbol{\delta}) k \\
\frac{d k}{s f(k)-(n+\delta) k}=d t \\
\int \frac{d k}{s f(k)-(n+\delta) k} d t=\int d t
\end{gathered}
$$

Se desconoce en general una antiderivada para la expresión $\frac{1}{s f(k)-(n+\delta) k}$ lo que imposibilita la solución analítica de la ecuación diferencial.

Para el caso en que la función de producción CES:

$$
\int \frac{d k}{s A\left(\alpha+(1-\alpha) k^{\rho}\right)^{1 / \rho}-(n+\delta) k}=\int d t
$$

En donde no se observa una solución de la integral en términos elementales. De igual forma la ecuación:

$$
{ }^{*}=s A\left(\alpha+(1-\alpha) k^{\rho}\right)^{1 / \rho}-(n+\delta) k
$$

No tiene una forma conocida dentro del estudio de la solución analítica de las ecuaciones diferenciales ordinarias de primer orden (a diferencia de las ecuaciones por variables separables). 
Esta es la razón principal por la que el uso de los métodos numéricos para la solución de ecuaciones diferenciales, tiene cabida en la ecuación fundamental de Solow con estas características.

Partiendo de un problema de valor inicial:

$$
\left\{\begin{array}{l}
y^{\prime}=f(x, y) \\
y\left(x_{0}\right)=y_{0}
\end{array}\right.
$$

Los métodos numéricos para la solución de ecuaciones diferenciales buscan determinar puntos aproximados a la solución analítica de la ecuación diferencial.

El método más simple, llamado Método de Euler, parte de la expansión en serie de Taylor (Kincaid \& Cheney, 1991):

$$
y(x+h)=y(x)+h y^{\prime}(x)+\frac{h^{2}}{2 !} y^{\prime \prime}(x)+\frac{h^{3}}{3 !} y^{\prime \prime \prime}(x)+\ldots+\frac{h^{n}}{n !} y^{(n)}(x)+\ldots
$$

$\mathrm{Al}$ truncar la serie desde el término $\frac{h^{2}}{2 !} y^{\prime \prime}(x)$ se obtiene la expresión:

$$
y(x+h) \approx y(x)+h y^{\prime}(x)
$$

Que permite dar una aproximación sobre el valor de la función $y(x+h)$ partiendo de la derivada (que corresponde a la ecuación diferencial) y el valor de la función en un valor anterior $y(x)$. Es importante aclarar que entre más pequeño sea el valor de , menor será el error producido por el truncamiento de la serie de Taylor de la función $y(x)$.

El método de Euler, al igual que otros métodos numéricos para la solución de ecuaciones diferenciales, son métodos iterativos basados en una fórmula que se ejecuta tantas veces como se desee. De esta forma se genera una sucesión de puntos que buscan aproximar la solución de la ecuación diferencial.

Para aplicar el método numérico empleamos la fórmula:

$$
y_{n+1}=y_{n}+h f\left(x_{n}, y_{n}\right)
$$

Donde $h$ es un valor dado y corresponde al paso deseado $(h$ se puede entender como la diferencia que hay entre $x_{k} \mathrm{y} \mathrm{x}_{\mathrm{k}+1}$. Esta fórmula parte del valor inicial $\left(x_{0}, y_{0}\right)$ y corresponde a un sistema de ecuaciones en diferencias de la forma:

$$
\left\{\begin{array}{l}
y_{n+1}=y_{n}+h f\left(x_{n}, y_{n}\right) \\
x_{n+1}=x_{n}+h
\end{array}\right.
$$

El proceso debe hacerse en un intervalo $[a, b]$ donde $a=x_{0}$ y $b$ es un valor definido previamente, que permite definir el intervalo donde se desea examinar la aproximación 
de la solución y debe ejecutarse un número de veces $M$ que corresponde al número de pasos que hay en el intervalo o hasta que se llegue al valor $b$.

Dado el problema de valor inicial, el algoritmo que puede seguirse es el siguiente:

Entrada: $h, b, x_{0}, y_{0}, M, f(x, y)$

Salida: $\left(x_{n}, y_{n}\right)$

Inicio:

Hacer: $x=x_{0} ; y=y_{0} ; n=1$

Mientras $x \leq b^{\wedge} n \leq M$ hacer:

$$
\begin{aligned}
& y=y+h f(x, y) \\
& x=x+h \\
& n=n+1
\end{aligned}
$$

Fin algoritmo.

Para emplear el método de Euler para aproximar la solución de la ecuación fundamental, es requerido el valor inicial $k(0)=k_{0}$ que corresponderá a la cantidad de capital per cápita de la economía en un momento del tiempo dado. La sucesión generada por el método, nos proporcionará el valor del capital en cada momento del tiempo antes de llegar al capital de estado estacionario.

\section{Solución numérica}

Para examinar diferentes alternativas de la solución numérica de la ecuación fundamental de Solow por el método de Euler, se supondrán los tres casos con: $s=0,3$; $\delta=0,1 ; n=0,015 ; A=1 ; \alpha=0,5$. Además se debe incluir un valor inicial que se tomará dependiendo del problema.

Caso 1: $\rho=0,6$

La ecuación fundamental de Solow corresponde a:

$$
k=0,3\left(0,5+0,5 k^{0,6}\right)^{1,667}-0,115 k
$$

El método de Euler fue programado en Mathematica ${ }^{\circledR}$ mediante el código siguiente:

ClearAll $[A, s, \alpha, k, \rho$, ahorrol, ahorro2, ahorro3, $t, a$,

ahorrol $=s A\left(\alpha+(1-\alpha) k^{\rho}\right)^{\frac{1}{\rho}} / \cdot\{s \rightarrow 0,3, A \rightarrow 1, \alpha \rightarrow 0,5, \rho \rightarrow 0,6\} ;$

ahorro $2=s A\left(\alpha+(1-\alpha) k^{\rho}\right)^{\frac{1}{\rho}} / \cdot\{s \rightarrow 0,3, A \rightarrow 1, \alpha \rightarrow 0,5, \rho \rightarrow 0,1\}$ 


$$
\begin{aligned}
& \text { ahorro } 3=s A\left(\alpha+(1-\alpha) k^{\rho}\right)^{\frac{1}{\rho}} / \cdot\{s \rightarrow 0,3, A \rightarrow 1, \alpha \rightarrow 0,5, \rho \rightarrow-3\} \\
& \text { NSolve }[\text { ahorrol }==0,115 k] \\
& M=200, h=10 ; \\
& a=\text { Table }[\{,\},\{n, 1, M / h+1\}] ; \\
& t=0, k=10 ; n=1 ; a[[1]][[1]]=0 ; a[[1]][[2]]=k ; \\
& W h i l e[t<M-1, a[[n]][[1]]=t ; k=k+h(\text { ahorrol }-0,115 k) ; t=t+h \\
& a[[n+1]][[2]] \\
& =k ; n++] \\
& a[[n]][[1]]=t
\end{aligned}
$$

Aquí se tiene en cuenta que el intervalo de tiempo es [0,200], y que además se tiene un valor inicial de $k(0)=10$. La solución aproximada del capital de estado estacionario corresponde a la línea: NSolve[ahorro1 $==0,115 \mathrm{k}$. Al ejecutar este comando se obtiene un valor aproximado de $k^{*}=31,974126228474134$.

Usando el método de Euler para aproximar la función del capital en función del tiempo se obtienen las siguientes parejas ordenadas:

Figura 4. Aproximación por Método de Euler con $\mathrm{M}=200 \mathrm{y} \mathrm{h}=10$

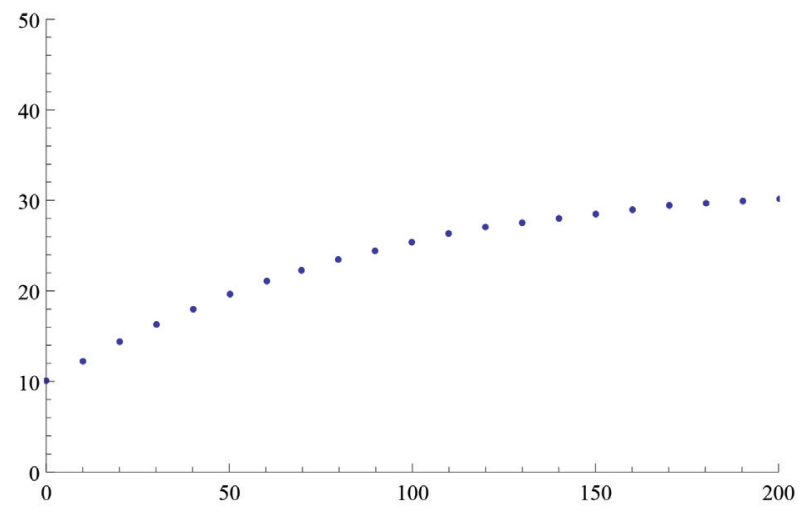


La última pareja iterada en este caso corresponde a $(200.30,25038)$. Sin embargo al generar una ampliación en el intervalo de tiempo, con $M=500$ se obtiene una última iteración de $(500.31,945)$ el cual corresponde a un valor más cercano al valor del capital de estado estacionario. En el caso que el intervalo de tiempo se duplica, se obtiene una última pareja correspondiente a (1000.31,97409537389806) obteniendo un error absoluto entre ellos de 0,000030854576074546, lo que sugiere que el método de Euler ha obtenido buen resultado al menos en generar una sucesión de puntos que converge al capital de estado estacionario.

Figura 5. Aproximación por Método de Euler con $M=1000$ y $h=10$

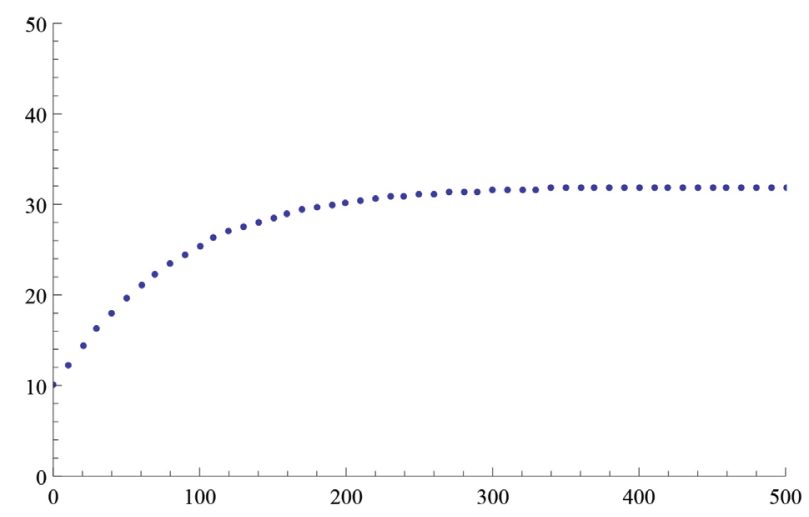

Caso 2. $\rho=0,1$

La ecuación fundamental corresponde a:

$$
\stackrel{*}{k}^{*} 0,3\left(0,5+0,5 k^{0,1}\right)^{10}-0,115 k
$$

En este caso, haciendo modificaciones al código en Mathematica ${ }^{\circledR}$, este no puede hallar una solución numérica para el capital de estado estacionario. Fue necesario entonces hallar una solución aproximada por el programa Derive6 ${ }^{\circledR}$ correspondiente a $k^{*}=7,534451207$.

Suponiendo un valor inicial $k(0)=40$, se obtiene la siguiente solución por el método de Euler: 
Figura 6. Aproximación por Método de Euler con y $M=1000$ y $h=10$

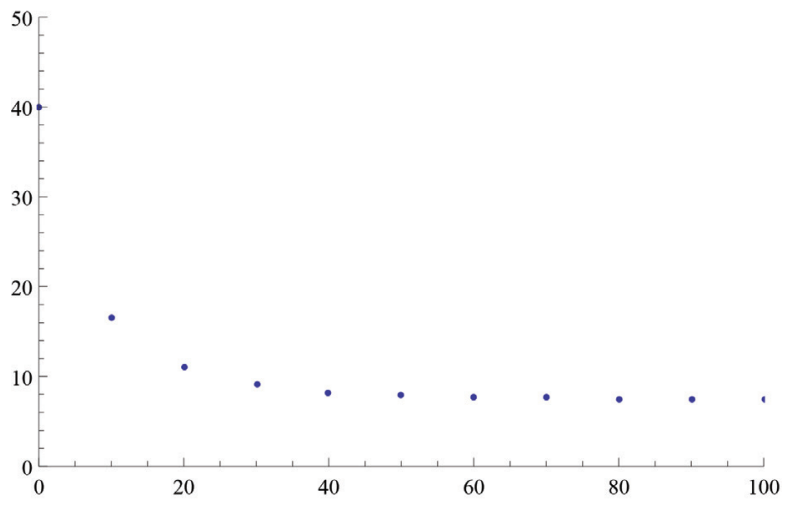

Cuya última iteración corresponde a (100.7,542742829). Al doblar el intervalo de tiempo se obtiene como última iteración (200.7,53445691737576). La cual genera un error absoluto de 0,00000571, obtenida en un menor intervalo de tiempo.

Caso 3. $\rho=-3$

La ecuación fundamental para este caso corresponde a:

$$
k=\frac{0,3}{\left(0,5+\frac{0,5}{k^{3}}\right)^{1 / 3}}-0,115 k
$$

Figura 7. Aproximación por Método de Euler con $M=200$ y $h=10$

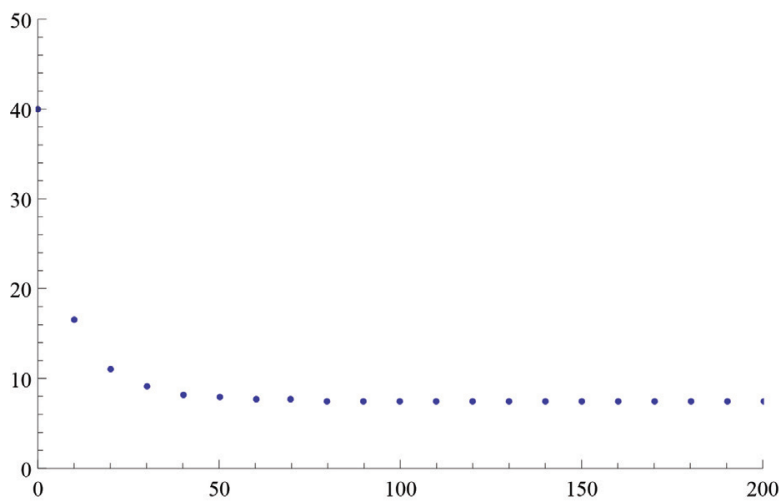

Empleando Mathematica ${ }^{\circledR}$ se obtiene una solución aproximada del capital de estado estacionario en $k^{*}=3,2555998621153823$. Suponiendo el mismo valor inicial del caso anterior $k(0)=40$ se obtiene la aproximación que se ilustra en la figura 8 . 
Al efectuar los cálculos se obtiene un último valor iterado de (100.3,2555999010262626). De lo que se obtiene un error absoluto de 3,891088029561729 $\times 10^{-8}$ en relación con el capital de estado estacionario.

Figura 8. Aproximación por Método de Euler con $M=100$ y $h=10$

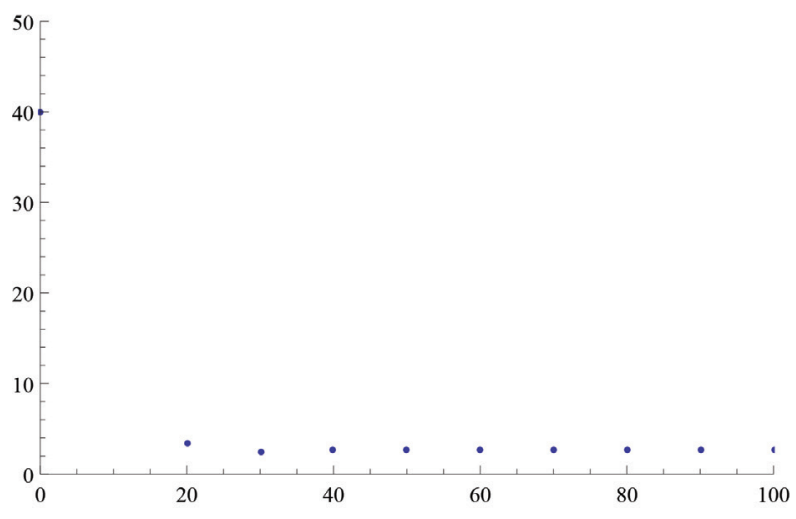

\section{Conclusiones}

Se observa un incremento importante en la complejidad de los procesos alrededor de la ecuación fundamental de Solow cuando la función de producción es CES, incluyendo la verificación de los supuestos de buen comportamiento. Las bondades que tiene la función CES en cuanto a su capacidad de contener formas más variadas para la producción son compensadas con las complejidades que genera su misma estructura.

De igual forma, al tratar de determinar el capital de estado estacionario, se encuentran no solo dificultades algebraicas en la existencia de soluciones reales positivas, sino dificultades en la solución numérica de la ecuación algebraica que se emplea para determinar $k^{*}$. En algunas ocasiones no se pueden de determinar soluciones mediante un software científico, y que aunque puede también emplearse algún método numérico para dicha tarea, puede considerarse como un aumento significativo en el estudio de la ecuación fundamental.

Puesto que se desconoce la solución analítica de la ecuación fundamental de Solow con una función de producción CES, el método de Euler logra establecer panoramas alentadores en este aspecto. Al tomar algunos valores de p de estudio, en ningún caso se puede descartar la convergencia de la función de capital al capital de estado estacionario. Matemáticamente corresponde a: $\lim _{t \rightarrow \infty} k(t)=k$.

Se observa también mediante los diferentes valores de intervalos de tiempo en el método de Euler, que entre menor sea el valor de $\rho$, menor tiempo empleará la economía para 
llegar al estado estacionario (el cual también es cada vez menor, en otras palabras, $\frac{\partial k^{*}}{\partial p}>0$ ). Para un caso en que con $\rho=-50$ con $M=100$, solo se requieren 10 iteraciones para llegar a un error absoluto de entre el valor aproximado del capital de estado estacionario con el valor aproximado de $k(100)$. Otros métodos numéricos más elaborados, como el método de Taylor de orden 4 o el método de Runge - Kutta de orden 4, permitirán obtener soluciones más aproximadas en menor tiempo de ejecución (Stoer \& Bulirsch, 1993).

De esta manera se logra concluir que los métodos numéricos para la solución de la ecuación fundamental de Solow, permiten obtener resultados que no se pueden obtener analíticamente cuando la función de producción no es tan agradecida como la función de producción Cobb - Douglas.

\section{Referencias}

Kincaid, D., \& Cheney, W. (1991). Numerical Analysis. Pacific Grove: Brooks/Cole Publishing Co.

Romer, D. (1996). Advanced Macroeconomics McGraw Hill.

Sala-i-Martin, X. (2000). Apuntes de Crecimiento Económico. Barcelona: Antoni Bosch Editor.

Stoer, J., \& Bulirsch, R. (1993). Introduction to Numerical Analysis. New York: Springer Verlag.

Sydsaeter, K., Strom, A., \& Berk, P. (2005). Economists' Mathematical Manual. Berlin: Springer. 
